\title{
Toward a highly sensitive polymer waveguide fiber Fabry-Pérot ultrasound detector
}

Supriya V. Thathachary

Shai Ashkenazi 


\title{
Toward a highly sensitive polymer waveguide fiber Fabry-Pérot ultrasound detector
}

\author{
Supriya V. Thathachary and Shai Ashkenazi* \\ University of Minnesota, Department of Biomedical Engineering, Minneapolis, Minnesota, United States
}

\begin{abstract}
In recent times, there has been extensive research on fiber-optic imaging devices in order to enable imaging/sensing at a size scale inaccessible to other modalities. The design for fabrication of a highly sensitive fiber-optic ultrasound detector is proposed. The transducer employs a polymer Fabry-Pérot resonator for ultrasound detection. To enhance acoustic sensitivity, a method is proposed for fabricating a self-aligned polymer waveguide within the cavity to improve the resonator quality factor ( $Q$-factor). Simulation studies were conducted to evaluate feasibility and quantify the improvement in $Q$-factor for different transducer configurations. Results show that with dielectric mirrors and a waveguide, $Q$-factor can approach the order of 10,000. Additional simulation studies are presented to analyze the effect of cavity shape on the device performance, drawing out the importance of a flat mirror for waveguided devices. Subsequently, results from optical testing of the first iteration of fabricated devices are presented, highlighting the main drawback of this method-the nonideal shape of the waveguiding pillar. Finally, initial results from the second iteration of devices that overcome this drawback are presented, demonstrating the feasibility of creating straight self-aligned polymer waveguides on gold-coated fibers, followed by a discussion on the implications of this work and future steps. @ 2018 Society of PhotoOptical Instrumentation Engineers (SPIE) [DOI: 10.1117/1.JBO.23.10.106008]
\end{abstract}

Keywords: intravascular imaging; Fabry-Pérot; fiber-optic; optical ultrasound detection.

Paper 180307R received May 31, 2018; accepted for publication Sep. 27, 2018; published online Oct. 27, 2018.

\section{Introduction}

The use of adjunctive imaging while attempting percutaneous coronary intervention (PCI) of chronic total occlusions (CTO) has been shown to reduce procedural complications such as arterial dissection, perforation, and cardiac tamponade. ${ }^{1}$ Renal biopsies are another example of a clinical procedure whose success rate could be significantly improved with tissue identification or imaging using a small scale forward-viewing device to differentiate between the cortex and medulla of the kidney. The literature suggests that tissue adequacy and safety are improved with guided-needle biopsies. ${ }^{2,3}$ Among several imaging modalities, intravascular ultrasound (IVUS) has been the most popular choice due to its high resolution and good penetration depth, ${ }^{4}$ and several studies have highlighted the efficacy of IVUS in characterizing ambiguous proximal caps, and in providing real-time far-field imaging to assist in CTO lesion crossing. ${ }^{5-8}$ Conventional IVUS transducers are cylindrical phased-array catheters, in which piezoelectric transducer arrays are mounted for side-looking imaging, ${ }^{9}$ however, studies highlight that forward-viewing transducers would be more suitable for guiding CTO PCI. ${ }^{10}$

In recent times, there has been a lot of research on all-optical ultrasound (US) transducers to overcome the difficulties involved with electrical connections needed for piezoelectric transducersa significant advantage considering the small size of catheters typically used to hold the transducer $(<1-\mathrm{mm}$ diameter). Optical transducers would also be able to overcome disadvantages, such as low signal-to-noise ratio and crosstalk, which are inherent to small size PZT devices. ${ }^{11}$ An additional advantage, significant from a commercialization standpoint, is that these optical ultrasound detectors are suitable for mass-manufacture, owing to the fabrication techniques, which would make them inexpensive to produce. Fiber-optic Fabry-Pérot interferometers, by virtue of their small size and high sensitivities at high frequencies, appear to be an ideal solution. ${ }^{12}$

Some research groups have successfully demonstrated such Fabry-Pérot US receivers fabricated on the tip of fiber-optic cables and in free space. ${ }^{13-15}$ Imaging using arrays of FabryPérot interferometer devices/mechanically scanning a singleelement device to simulate an array has also successfully been demonstrated. ${ }^{16,17}$ Fiber-based devices have an advantage over free space due to the ease in delivering excitation light to the area of interest-either as light itself (for photoacoustic imaging) or in the form of ultrasound pulses (by exciting certain materials to produce ultrasound waves by thermoelastic ultrasound generation). ${ }^{18}$ For a resonator with small mirror areas, lateral losses due to beam walk-off as a result of multiple reflections at nonnormal incidence become significant. These losses reduce the overall energy of the interferometer, which is equivalent to reduced reflectivity of the mirrors, and cause phase dispersion due to increased path lengths. Therefore, the quality factor ( $Q$-factor) of the resonator is significantly reduced. Li et al. ${ }^{19}$ proposed a concave cavity, which confines the beam laterally, as a solution to this problem. A fiber-optic transducer and results from imaging via a raster scan using this type of plano-concave Fabry-Pérot cavity have also been demonstrated to have noise equivalent pressure (NEP) on the order of 10 to $50 \mathrm{~Pa}^{20}$ Their technique relies on increasing $Q$-factor by controlling the curvature of the resonator to exactly match the emerging wave front to refocus the reflected light back into the fiber. This curvature control, in turn, is dependent on 
the surface tension of the polymer medium used to create the plano-concave device and results in a certain fixed thickness for a given base diameter. This restricts the possibility of scaling the device thickness to optimize the device with respect to bandwidth. Extending this technology to fiber bundles also presents significant challenges due to the need for precise volume and alignment control while depositing the polymer layer onto each fiber in order to obtain a curvature that exactly matches the emerging wave front.

This paper proposes a method to improve the $Q$-factor of the Fabry-Pérot detector by providing lateral confinement on a fiber-optic device by writing a self-aligned waveguide into the polymer in the resonating cavity. This method relaxes the requirement on matching the curvature to the emerging wave front, allowing for scalability in thickness to improve sensitivity at bandwidths of choice. Tadayon et al. demonstrated a large size (1" diameter) multimode device employing a step-index profile to reduce lateral losses, in which cylindrical pillars of SU-8 2010 polymer (refractive index: 1.67) were created using photolithography, and a cladding of thiol-ene/methacrylate photopolymer (TM polymer) with 1.53 refractive index was deposited around the pillars. This step-index profile enables total internal reflection within the resonating cavity in a manner similar to optical fibers, ${ }^{21}$ thus improving optical finesse from 40 to 200. This paper presents a method to create such a self-aligned polymer waveguide on a fiber thereby achieving high sensitivity at the size scale of a fiber device. Using such a fiber Fabry-Pérot interferometer device with a self-aligned waveguide, a higher $Q$-factor can be achieved leading to higher acoustic sensitivity.

Such a fiber Fabry-Pérot ultrasound device, given its small size $(<250 \mu \mathrm{m})$, could be embedded inside standard guidewires used in interventional cardiology, or within biopsy needles, to enable photoacoustic tissue differentiation. Tissue differentiation and identification of vulnerable plaque using photoacoustic imaging have widely been demonstrated. ${ }^{22-24}$ Therefore, this device could either be used in conjunction with a scanning mechanism for imaging, or for sensing applications to identify and differentiate tissues during CTO PCI or biopsies. This paper describes the simulation studies used to optimize the design of such a device, initial experimental results demonstrating feasibility of fabricating self-aligned polymer waveguides, and, with the support of simulations, lays down the plan for fabrication of the complete device.

\section{Proposed Transducer}

\subsection{Principle of Device}

A Fabry-Pérot interferometer consists of a resonating cavity formed between two mirrors and an optically transparent medium between them, as shown in Fig. 1(a). Part of the light incident on the mirror gets reflected repeatedly between the two mirrors, with a periodic resonance condition occurring at every wavelength, for which the thickness of the cavity is equal to an integral number of effective half wavelengths (absolute wavelength divided by the refractive index). To detect the ultrasound wave, the intensity of reflected light over a range of interrogation wavelengths is studied, and the value corresponding to the highest slope is chosen for maximum sensitivity. Under interrogation using this optimal wavelength, when the acoustic pressure wave hits the cavity, it changes the thickness of the cavity and consequently the wavelengths, in which optical resonances occur. This results in a change in the intensity of the received light at the tuned wavelength, and thus the ultrasound wave can be detected.

\subsection{Fabry-Pérot Cavities: Effect of Waveguide on Q-Factor}

For an ideal resonator, both resonator mirrors are unconfined and perfectly reflecting, and no power is lost during the round trip. However, for a practical resonator with small mirror areas, lateral losses due to multiple reflections at nonnormal incidence become significant, as illustrated by $\mathrm{Li}$ et al. ${ }^{19}$ These losses reduce the overall energy of the interferometer, which is equivalent to reduced reflectivity of the mirrors, and also cause phase dispersion due to increased path lengths. Therefore, the $Q$-factor of the resonator is significantly reduced. This loss can be compensated for in two ways by: increasing the reflectivity of the mirrors and restricting the diffraction loss in the lateral direction by introducing a waveguide in the resonator cavity. A waveguide can be created in the cavity by inducing a small refractive index change in the resonator medium to create a step-index profile. The following sections describe simulation studies carried out to study the effect of waveguides on $Q$-factor in cavities of different thicknesses to decide the optimal thickness, and the fabrication and testing of a prototype waveguide device. Thereafter, further refined simulation models have been created to study the effect of various physical
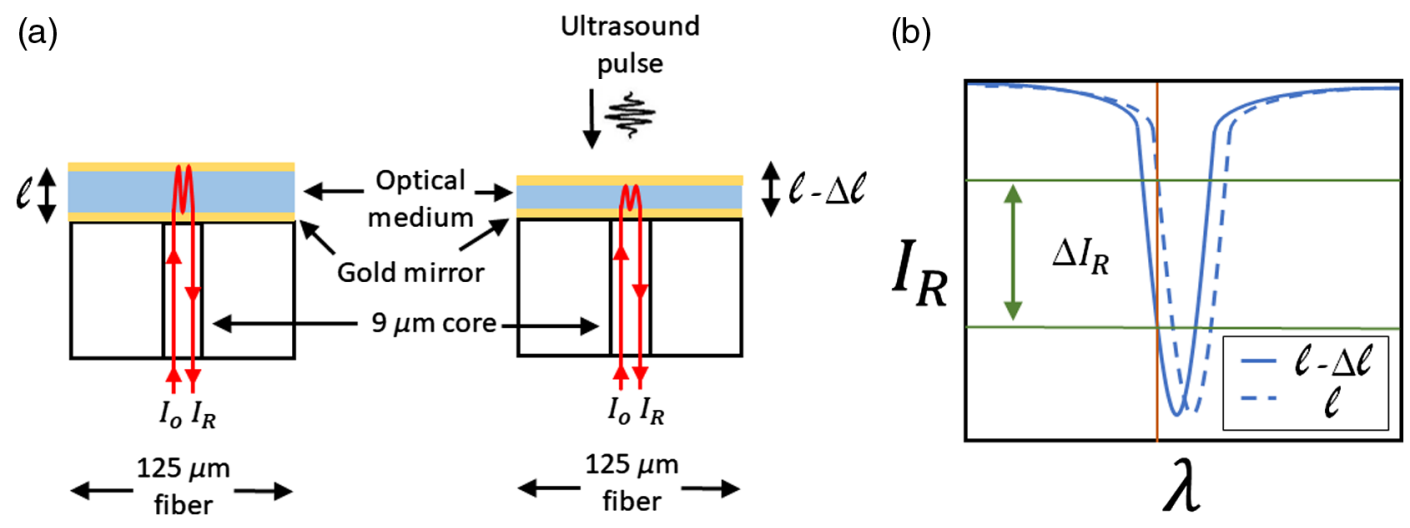

Fig. 1 Fabry-Pérot ultrasound detector: (a) structure of the device showing NIR wavelength interrogation and (b) shift in resonance upon ultrasound detection. 
parameters on the optical performance with a view to use the results from these simulations to guide further development and optimization of the fabricated device.

\subsection{Optical Resonator Q-factor and Acoustic Sensitivity}

An important metric to evaluate the performance of an ultrasound detector is its NEP, the smallest detectable pressure signal for the device. For a Fabry-Pérot resonator, this minimal detectable pressure is inversely proportional to the resonator finesse $F^{25}$

$\mathrm{Pac}_{\min }=\sqrt{\frac{4 q B}{27 S I_{0}}}\left(\frac{\lambda}{F n}\right) \frac{Y}{L}$,

where $q$ is the electron charge in Coulombs, $B$ is the bandwidth of the photodetector in $\mathrm{Hz}, S$ is the optical detector sensitivity in $\mathrm{A} / \mathrm{W}, I_{0}$ is the incident optical beam power, $\lambda$ is the optical wavelength in vacuum, $n$ is the refractive index of the resonator medium, $Y$ is the Young's modulus of the medium, and $L$ is the cavity length under rest. Finesse itself is defined as

$F=\frac{\Delta \nu}{\delta \nu}$

where $\Delta v$ and $\delta v$ are the free spectral range (FSR) and full width half maximum of the resonance peak in frequency, respectively. ${ }^{26}$ Rewriting the $\lambda / F$ term from Eq. (1) in terms of frequency and substituting for finesse from Eq. (2), we get:

$\mathrm{Pac}_{\min }=\sqrt{\frac{4 q B}{27 S I_{0}}}\left(\frac{c \cdot \delta \nu}{\Delta \nu \cdot \nu_{0}}\right) \frac{Y}{L n}$

Resonator $Q$ factor can be defined as

$Q=\frac{\nu_{0}}{\delta \nu}$,

where $\nu_{0}$ is the frequency, in which the resonance peak occurs. Additionally, the FSR is defined as

$\mathrm{FSR}=\Delta \nu=\frac{c}{2 n L}$

Therefore, using Eqs. (4) and (5), we can rewrite Eq. (3) as

$\mathrm{Pac}_{\min }=\sqrt{\frac{4 q B}{27 S I_{0}}} \frac{2 Y}{Q}$.

This demonstrates that a higher $Q$-factor for the optical resonator implies a lower NEP and, consequently, a greater acoustic sensitivity. In this paper, therefore, the resonator $Q$-factor has been used as the performance metric to optimize the sensor design.

\section{Simulation Studies}

As described in the previous section, the device will consist of a resonating cavity between two mirrors. Because of its influence on sensitivity, the $Q$-factor of the resonator is one of the most important design criteria around which the device would be optimized. Considering the structure of the device, it was important to account for the following effects that negatively impact the $Q$-factor of the resonator:

(a) The beam broadens as it propagates within the cavity, which causes non-normal incidence on the second mirror, and in all subsequent reflections. Due to these successive reflections between the mirror at non-normal incidence, there is a loss in the power coupled back into the fiber core, i.e., power losses in the lateral direction.

(b) Any loss of energy due to imperfect mirror reflection is exacerbated because of the multiple successive reflections occurring within the cavity.

Therefore, prior to fabricating the device, simulation studies were carried out on the Rsoft Design Suite software with the view to understand the impact of these losses on $Q$-factor and the best device design for minimizing them.

\subsection{Waveguide to Address Diffraction Losses}

In order to visually demonstrate the efficacy of a waveguide in restricting beam walk off upon transmission over a distance corresponding to several round trips, a simulation study was set up. This simulation has been performed over a thickness of $1000 \mu \mathrm{m}$ even though the envisaged final device thickness is only $\sim 20 \mu \mathrm{m}$ in order to understand and compare the beam divergence for the two cases that would result after multiple round trips within the resonator. The refractive index of the material within the cavity was set to 1.5 (comparable to glass). The optical field in the polymer was studied to understand the distribution of energy. As seen in Fig. 2(a), the near-infrared (NIR) field spreads in the polymer to a diameter of around $100 \mu \mathrm{m}$ at an axial distance of $800 \mu \mathrm{m}$ from the source.

A second simulation was done to evaluate the effectiveness of including the waveguide in containing the field distribution. This was done by selectively modifying the refractive index of the waveguide to mimic the core and cladding of the fiber (1.506 and 1.500 , respectively). The results of this simulation are seen in Fig. 2(b). Without a waveguide, there is significant lateral loss in power over a transmission of $1000 \mu \mathrm{m}$. In the case of the device with waveguide, for a distance of $1000 \mu \mathrm{m}$ (25 round trips for a $20-\mu \mathrm{m}$-thick device), the power is still restricted within the $9-\mu \mathrm{m}$ core, proving the efficacy of the waveguide in reducing lateral losses.

A subsequent simulation of the full device with real dimensions is presented in Fig. 3, which shows a quantitative confirmation of the same effect via improvement in $Q$-factor. The values of the refractive indices assigned to the waveguide and surrounding portions of the polymer were 1.506 and 1.500 , respectively. A $45-\mu \mathrm{m}$ cavity was simulated between two gold mirrors of 30-nm thickness with and without a waveguide. The corresponding optical resonances confirm that the $Q$-factor was seen to improve significantly-from 1853.33 without a waveguide to 3173.04 after including a waveguide.

Since beam divergence increases with higher thickness, it was expected that the improvement with including a waveguide would be more drastic for thicker devices. To confirm this, a study was done comparing the improvement with the introduction of a waveguide for three different Fabry-Pérot interferometer thicknesses, 20, 45, and $80 \mu \mathrm{m}$. These thicknesses correspond to FSR of 40, 17, and $10 \mathrm{~nm}$, respectively. As 

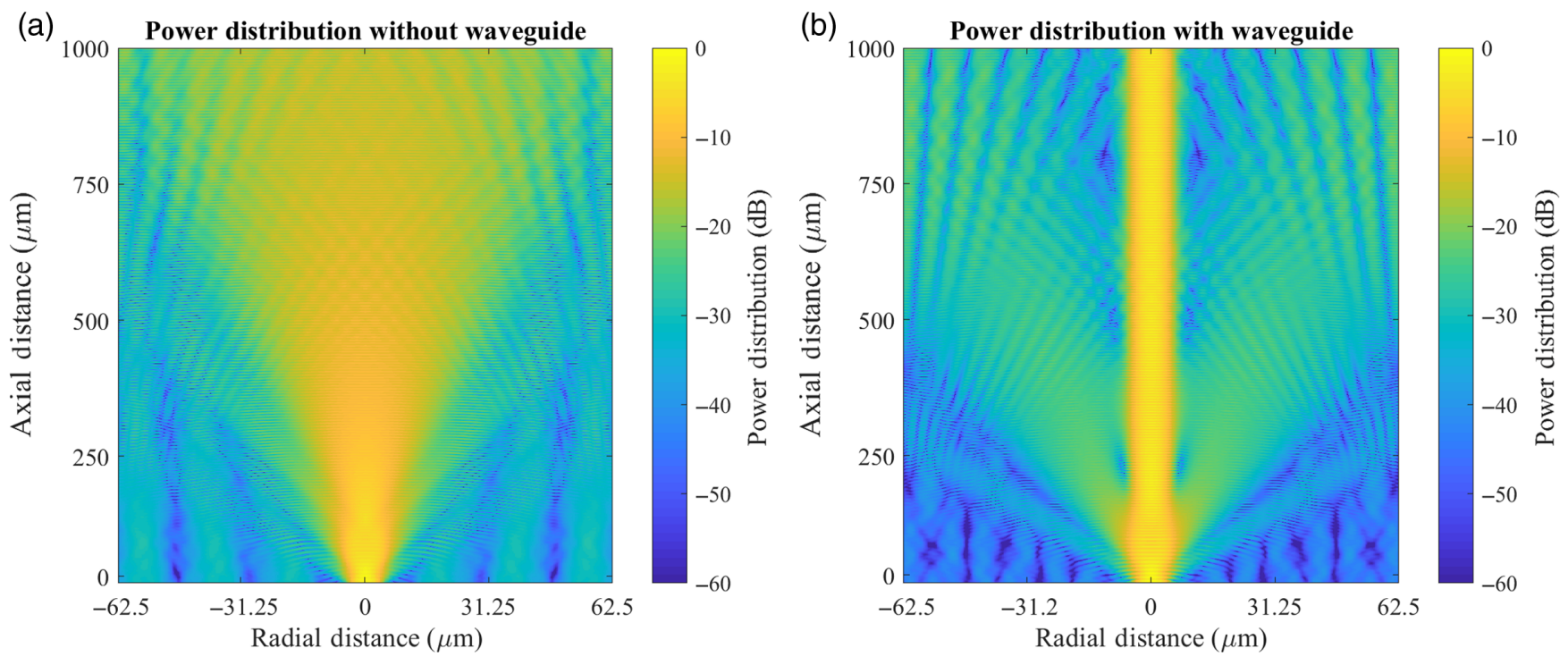

Fig. 2 Simulation results showing power distribution in $d B$ for Fabry-Pérot interferometer devices (a) without a waveguide and (b) with waveguide.

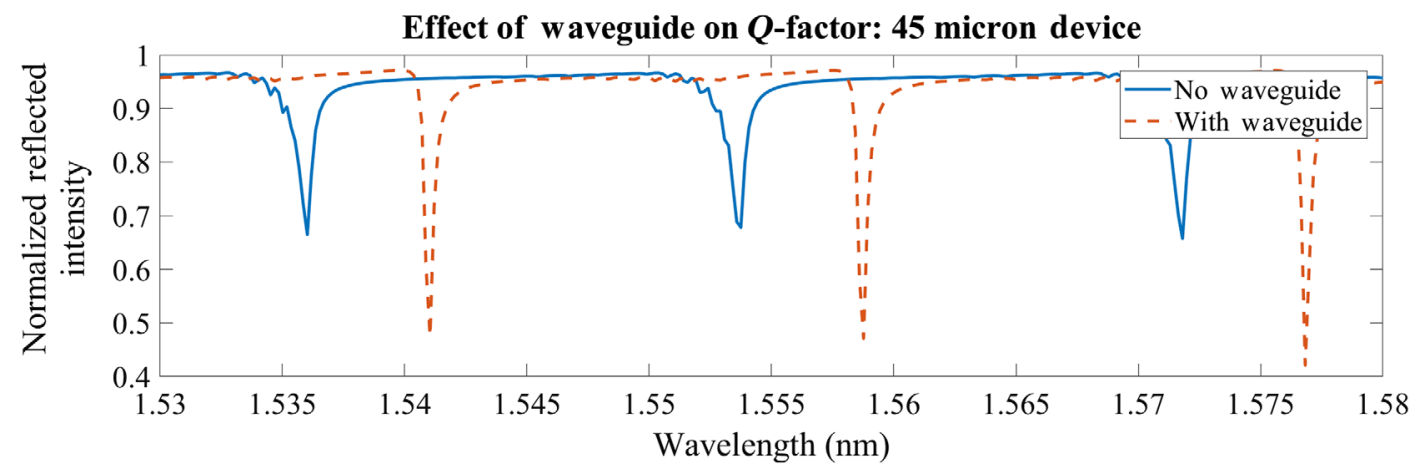

Fig. 3 Improvement in Q-factor of the resonator upon introduction of waveguide.

expected, the improvement in $Q$-Factor with the presence of a waveguide is seen to increase with cavity thickness (Fig. 4).

The improvement in $Q$-factor upon the introduction of the waveguide was highest for the $80-\mu \mathrm{m}$ device, from 3034.01 to 4779.94 . In the case of the $45-\mu \mathrm{m}$ device, the improvement was reduced, and the $Q$-factor changed from 1853.33 to 3173.04. The least improvement, from 1301.04 to 1403.46 , was observed in the case of the $20-\mu \mathrm{m}$ device. These simulations served as a confirmation that a self-aligned waveguide would provide significant improvement to the $Q$-factor of the device (Table 1).

\subsection{Effect of Mirror Reflectivity on Q-Factor}

In the work discussed in the previous section, the mirrors for the resonating cavity were 30-nm gold layers. Gold was chosen as a mirror for the preliminary design because of the ease of fabrication, sufficient reflectivity at NIR wavelengths (>95\%) used for interrogation of the cavity, and poor reflectivity at UV wavelengths, which need to penetrate the gold layer to cure the epoxy - the optical medium of the resonator. However, since there are multiple successive reflections, there is significant energy lost from the cavity even with $95 \%$ reflectivity offered by the gold mirrors. Since the finesse, and therefore, the $Q$-factor of the resonator depend on mirror reflectivity, ${ }^{25}$ the $Q$-factor for devices with gold mirrors was not very high. This can be improved using dielectric Bragg mirrors instead.

Dielectric mirrors consist of multiple thin layers of (usually two) different transparent optical materials and offer very high reflectivity (above 99\%) because the reflections from the multiple interfaces constructively interfere. Bragg mirrors comprise several dielectric layers, each of which has a thickness equal to quarter of the design wavelength. This design leads to the highest possible reflectivity for a given number of layer pairs and given materials. Tadayon et al. ${ }^{21}$ used similar Bragg mirrors in their Fabry-Pérot interferometer in large size multimode devices, consisting of eight quarter-wavelength layers of titanium dioxide $(n=2.19)$ and seven quarter-wavelength layers of silicon dioxide $(n=1.46)$, designed for high reflectivity at $1550 \mathrm{~nm}$.

A $45-\mu \mathrm{m}$ thick cavity simulated using this design showed an improved $Q$-factor of 7855.96 as compared to 2095.32 with gold mirrors, as seen in Fig. 5. Including a waveguide structure in addition to the dielectric mirrors further improved the $Q$-factor of the device to 10827.66 . This is especially significant as it implies that high $Q$-factors may be achieved even at lower thicknesses, which allows for greater acoustic bandwidths. 

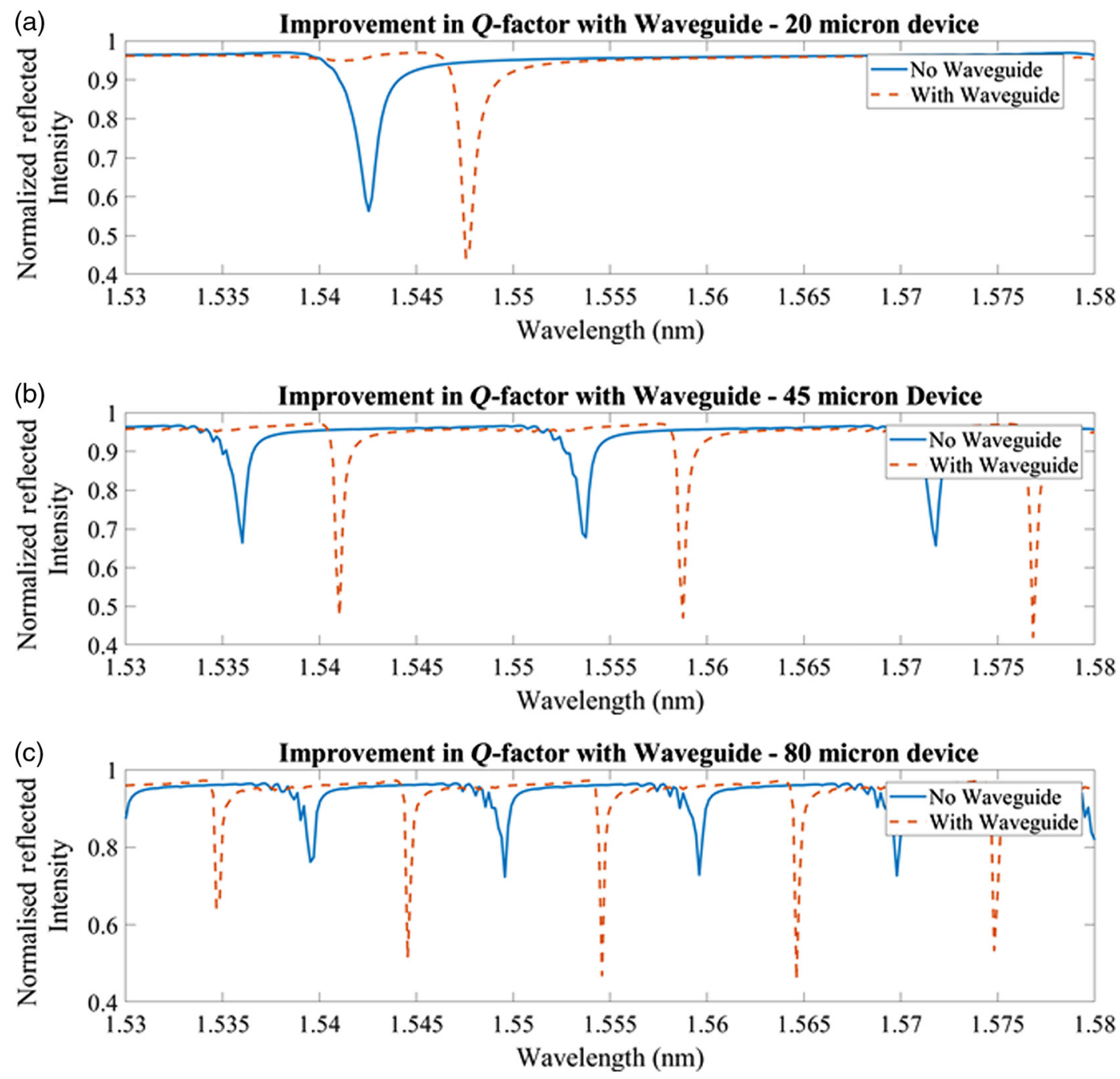

Fig. 4 The improvement of $Q$-Factor with the presence of a waveguide for different cavity thicknesses.

Table 1 Improvement in $Q$-factor upon the introduction of the waveguide for different thicknesses.

\begin{tabular}{lccc}
\hline Device thickness & $80 \mu \mathrm{m}$ & $45 \mu \mathrm{m}$ & $20 \mu \mathrm{m}$ \\
\hline Change in Q-factor & 3034.01 to & 1853.33 to & 1301.04 to \\
& 4779.94 & 3173.04 & 1403.46 \\
\hline
\end{tabular}

\subsection{Planar Versus Plano Concave Simulation Models}

The planar structure described in Secs. 3.1 and 3.2 provides a simplified model to test the improvement in $Q$-factor upon the inclusion of a waveguide. However, this does not accurately describe the structure fabricated on the tip of a single-mode fiber. Dip-coating of the epoxy would result in a plano-concave structure due to surface tension holding the epoxy on the fiber tip surface. In order to accurately model this and understand the effect of cavity shape on the device performance, the planar simulation model was studied in comparison to plano-concave structures. The largest contact angle possible for a planoconcave device would be $90 \mathrm{deg}$, resulting in a hemispherical drop. For lower contact angles, a gentler curvature on the droplet

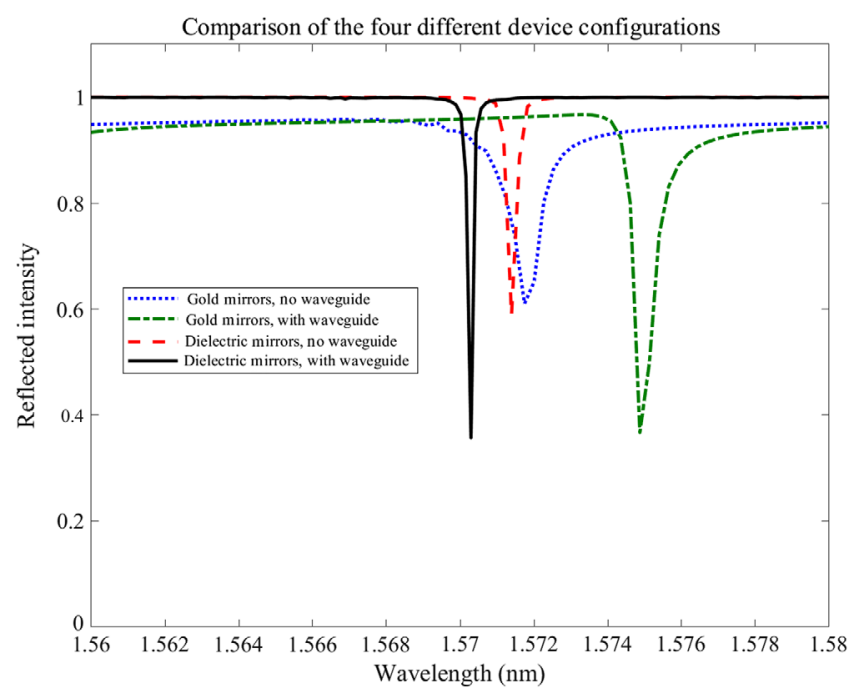

Fig. $5 Q$-factor enhancement with waveguides and dielectric mirrors.

can be expected. Figure 6 shows a comparison of the optical characteristics of planar and hemispherical devices plotted alongside the focused plano-concave cavity created with the dimensions of the device presented by Guggenheim et al. ${ }^{20}$ 
(a)

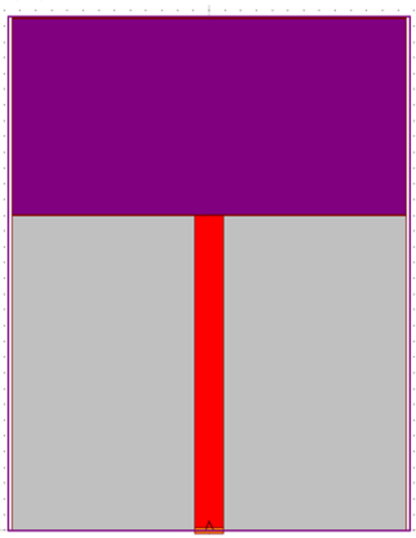

(b)

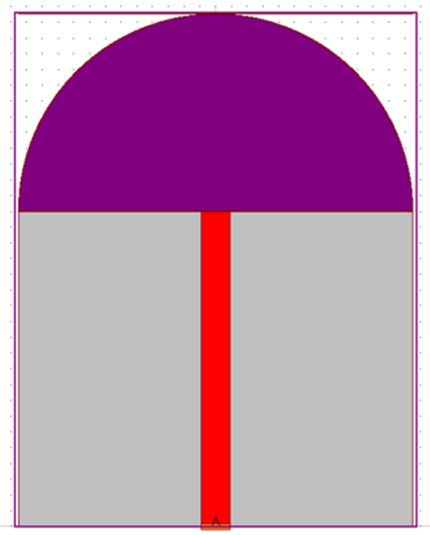

(c)

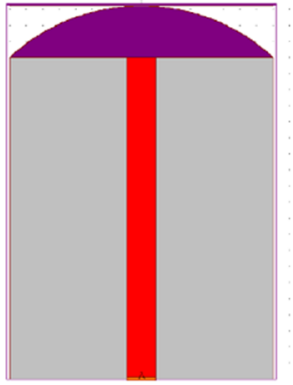

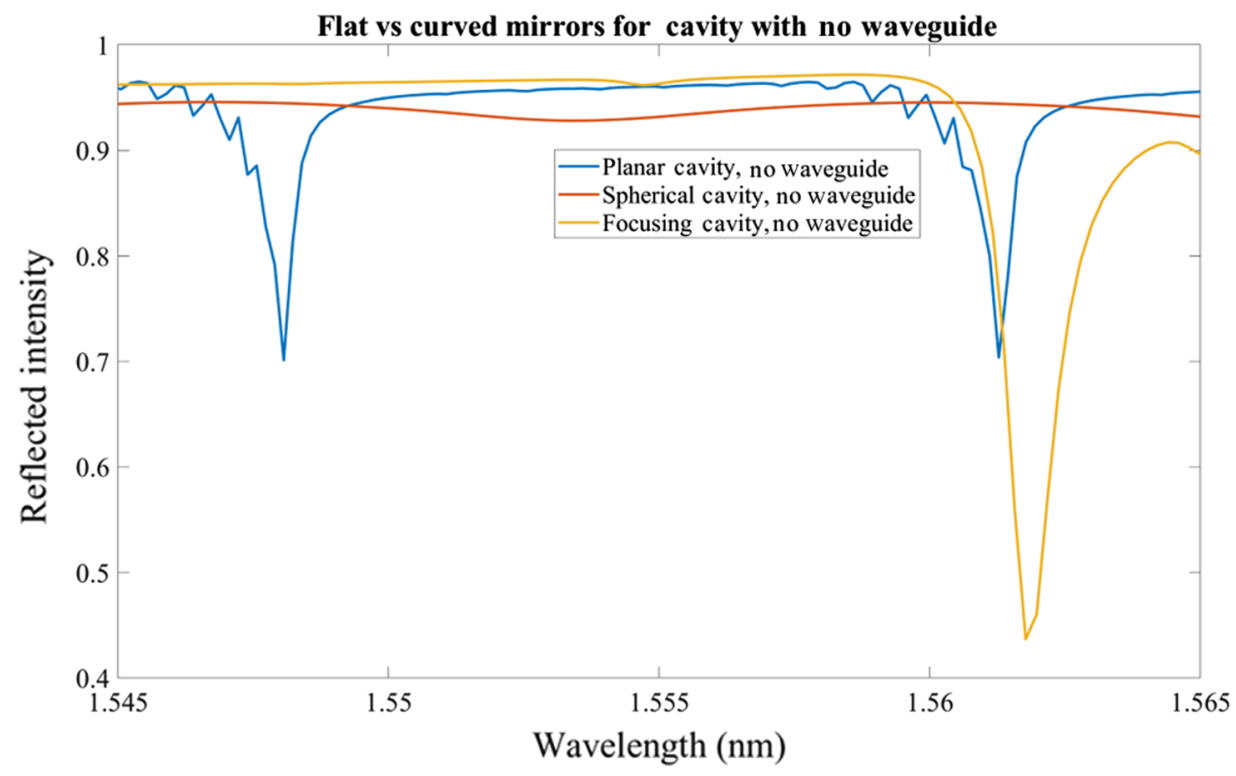

Fig. 6 Comparison of characteristic curves of (a) planar, (b) hemispherical, and (c) focusing resonator structures.

The total height of the planar and hemispherical devices was kept the same for a fair comparison.

The hemispherical device showed a negligible $Q$-factor of 387.18 (finesse of 3.22) in comparison to the planar cavity with $Q$-factor of 3123.17 and finesse of 26.23 for the planar device. This difference is because the hemispherical cavity is unable to focus light effectively back into the fiber cavity. The focused plano-concave model, on the other hand, can do so most effectively as its curvature exactly matches the emerging wave front. This approach has been used to improve finesse by a factor of 4 as compared to planar devices. ${ }^{27}$ An important difference in our approach, however, is the presence of a waveguide-implying a flat wave front as opposed to the divergent beam that would be present in a device without a waveguide. This dictates that we require the second mirror to be as flat and parallel to the first mirror as possible for optimal performance. In order to understand the effect of the device curvature in the case of waveguided devices, a second set of simulations was performed, comparing waveguided hemispherical and planar cavities, presented in Fig. 7.

As expected, the performance of the planar cavity surpassed that of the hemispherical cavity as the flat second mirror is better suited to reflect the plane wave front that emerges from the waveguide.

\subsection{Plano-Concave Resonators with Gentle Curvature Approaching Flat Mirrors: the Fiber-Ferrule Device}

Although it is possible to control the curvature of the concave second mirror to an extent using epoxies with different contact angles, it is difficult to achieve the flat parallel resonator structure required for optimal performance of the waveguided device on a bare fiber. One way to achieve this is to insert the fiber into a ferrule and use the larger surface area to deposit a flat parallel mirror. This approach was evaluated through simulations using a model based on dimensions resulting from experimental measurements of dip-coating using a fiber-ferrule assembly (described in Sec. 4.3). The distance between the tip of the pillar and the second mirror can be varied depending on how far beyond the surface of the ferrule the fiber is placed and secured. To determine to what extent this positioning might affect the device $Q$-factor, and therefore, the tolerance on the position of the fiber with respect to the ferrule, a set of simulation 
(a)

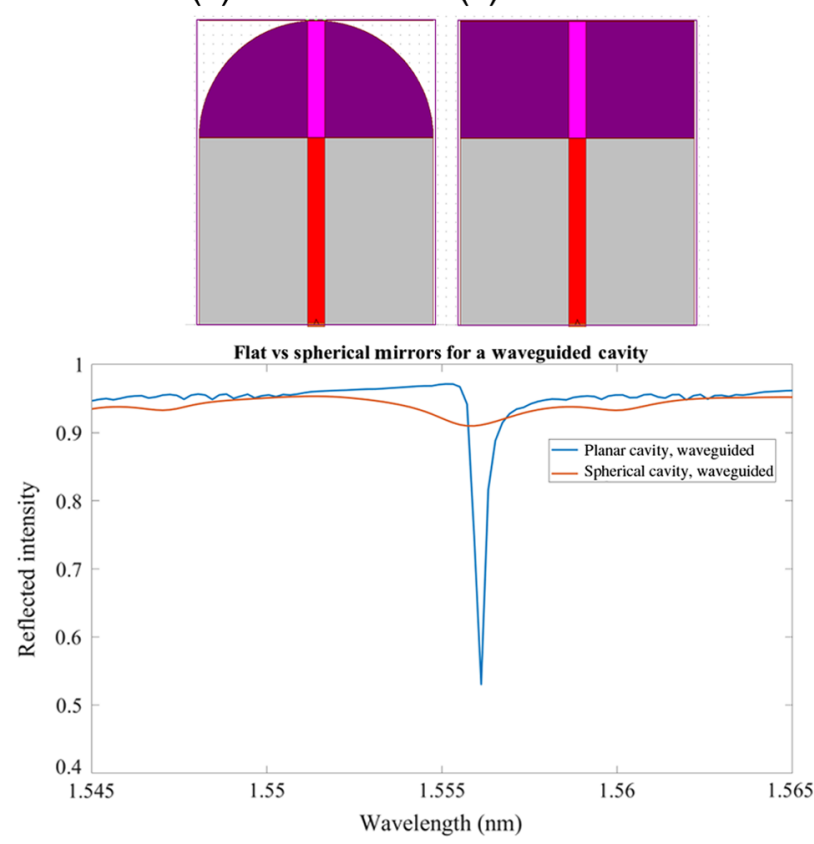

(b)

Fig. 7 Characteristic curves of hemispherical and planar waveguided cavities illustrate the importance of having a flat mirror for waveguided cavities. models were designed to scale (for the portion of the device demarcated in Fig. 13) using the contact angle of 37 deg from the experiment described in Sec. 4.3, and the ferrule surface of diameter $1.8 \mathrm{~mm}$.

As shown in Fig. 8, it is observed that the distance between the tip of the fiber and the second mirror significantly affects the $Q$-factor. The $Q$-factor for the device without a waveguide was 1558.30 (finesse 14.11), and those of the devices with waveguides that were $75 \%, 85 \%, 95 \%$, and $100 \%$ of the total height of the device were 3113.59 (finesse 26.20), 3916.51 (finesse 32.22), 5255.45 (finesse of 39.79), and 5205.40 (finesse 40.40), respectively. From this study, it was inferred that the fiber must be positioned above the ferrule at a position such that the waveguide structure is at least $90 \%$ of the total device height. Running this test also provides us with the best estimate for the $Q$-factor of a device fabricated using this approach. As discussed in Sec. 3.2, dielectric mirrors in place of the gold mirrors used would result in a significantly higher $Q$-factor.

\section{Fabrication}

\subsection{Bare Fiber Device Fabrication}

The first iteration of devices fabricated consisted of planoconcave Fabry-Pérot interferometers constructed on singlemode optical fibers $125-\mu \mathrm{m}$ diameter. The following steps
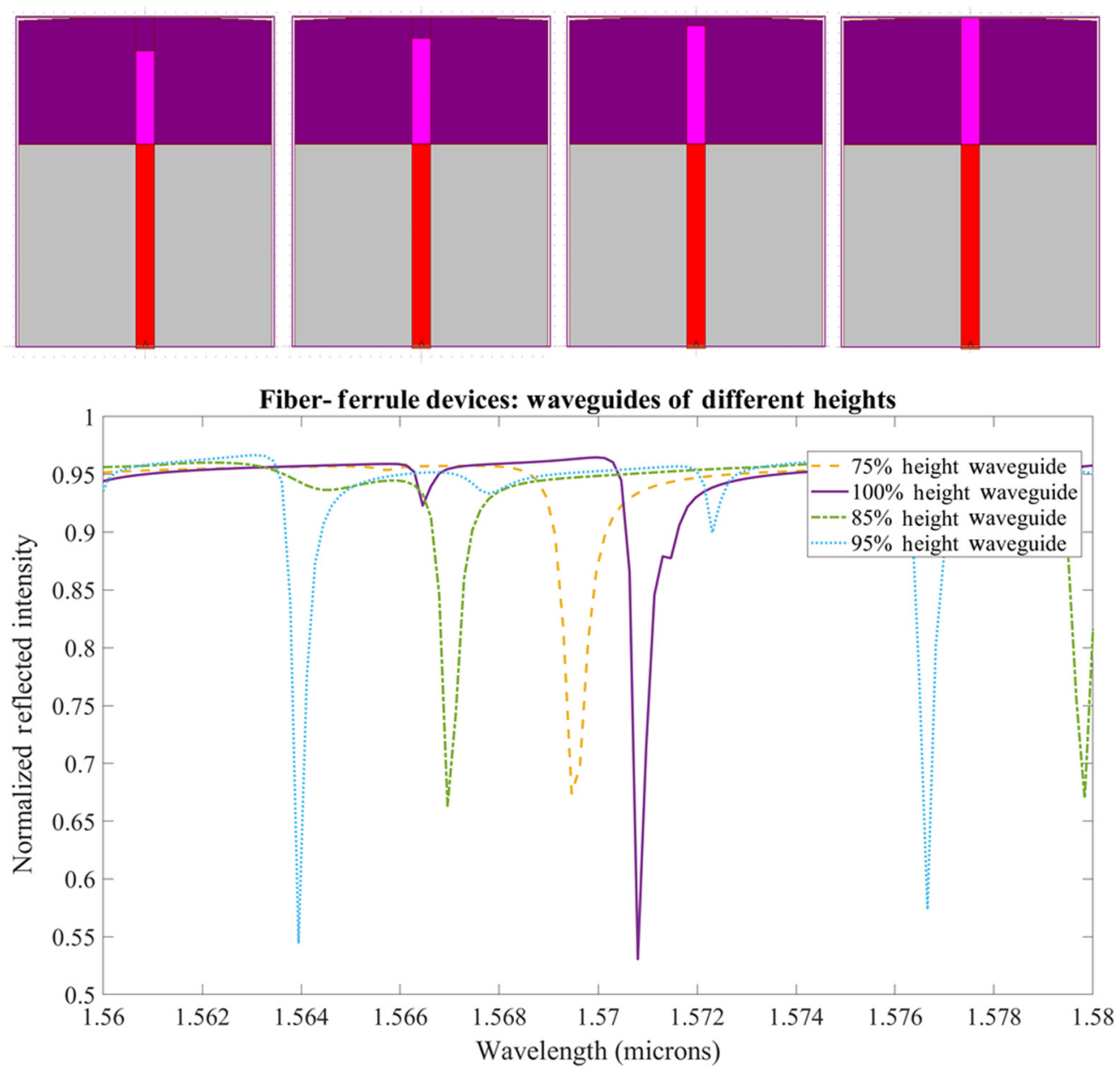

Fig. 8 Simulations run to estimate the tolerance on distance of the fiber tip from the second mirror and the associated characteristic curves. 
(a)

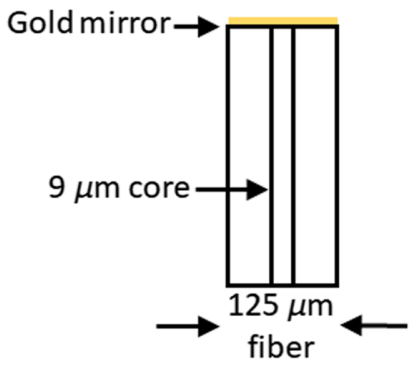

(d)

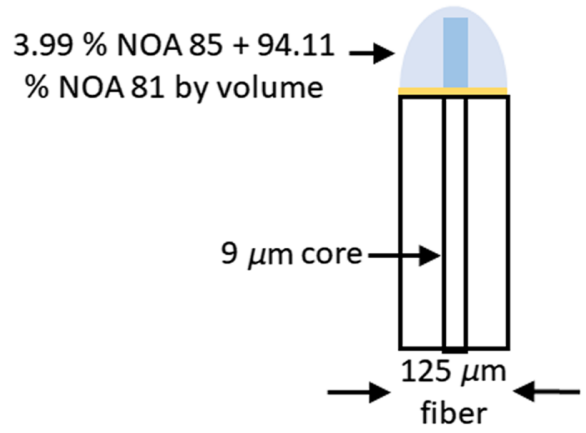

(c)

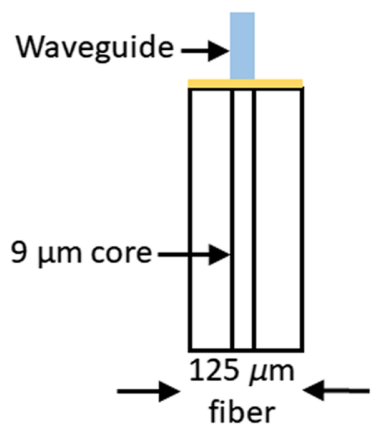

(e)

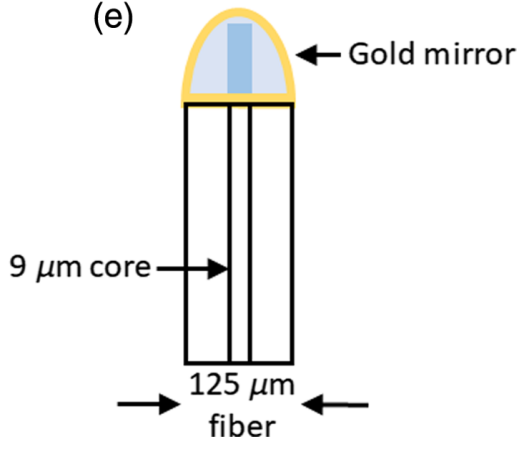

Fig. 9 Fabrication steps for fiber device: (a) first mirror deposited on fiber, (b) dip coating in waveguide epoxy, (c) pillar obtained by selective curing, (d) dip coating in cladding epoxy, and (e) deposition of second mirror.

were followed for fabrication, as presented in our earlier work: $:^{28}$

1. The single-mode optical fiber substrate with $9-\mu \mathrm{m}$ core and $125-\mu \mathrm{m}$ cladding was stripped and cleaved to produce an optically smooth surface.

2. A 30-nm gold layer was deposited on the polished surface by e-beam evaporation, forming the first mirror of the resonator, as shown in Fig. 9(a).

3. The gold-coated fiber tip was then dipped into a vial of UV curable epoxy Norland Optical Adhesive (NOA) 81 having refractive index 1.56 . The speed of immersion and retraction as well as the depth of immersion were controlled so as to obtain a droplet on the fiber tip, as shown in Fig. 9(b).

4. The droplet was exposed to a UV lamp (Omnicure S1000) having wavelength range of 320 to $500 \mathrm{~nm}$. The exposure was done through the fiber connector, thus selectively curing only the portion of epoxy directly above the core. The fiber tip, along with the partially cured droplet, was then washed in acetone, resulting in an epoxy pillar as pictured in Fig. 9(c). A microscope image of the same is seen in Fig. 10(a).

5. A mixture of epoxies NOA 85 and NOA 81 was made choosing the rations of the two polymers to achieve an effective refractive index 1.556. The fiber tip containing the pillar was then dipped in this mixture to create the cladding of the device, as seen in Fig. 9(d).

6. This cladding was flood exposed to UV from the front side, thus curing the entire droplet, with the pillar of higher refractive index embedded at its center. A second 30-nm gold layer was then deposited, completing the resonator, as seen in Fig. 9(e). A microscope image of the completed device is seen in Fig. 10(b).

As seen in Fig. 10(a), the wave-guiding pillar was not straight and flat as desired but rather had an irregularly shaped tip, which would adversely affect the performance of the device by distorting the emerging wave front. Experiments conducted to further understand the cause for this are presented in subsequent sections. The completed device was then tested for optical resonance and the results are presented below.

\subsection{Results from Testing}

\subsubsection{Testing for optical resonance}

The completed device was then tested for optical resonance on a tunable NIR laser of wavelength range 1510 to $1640 \mathrm{~nm}$ via a fiber circulator. Results are as shown in Fig. 11. The top graph shows results obtained on a device manufactured without a waveguide. In this case, the FSR is $2386 \mathrm{GHz}$. This corresponds to a droplet height of $40.29 \mu \mathrm{m}$. The $Q$-factor and finesse of (a)

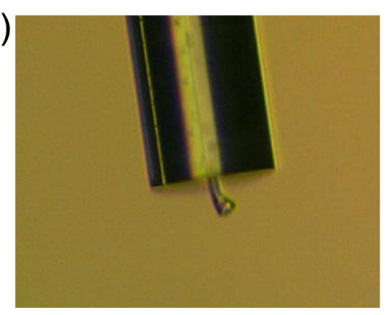

(b)

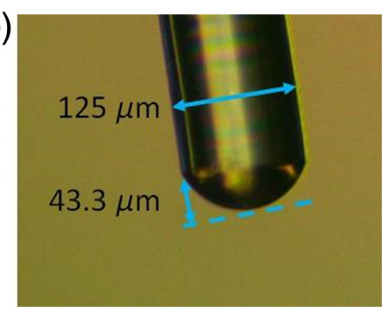

Fig. 10 Microscope images of the device (a) after fabrication of the wave-guiding pillar and (b) after the dip-coating of the surrounding epoxy. 
a) Characteristic curve for fiber device without waveguide

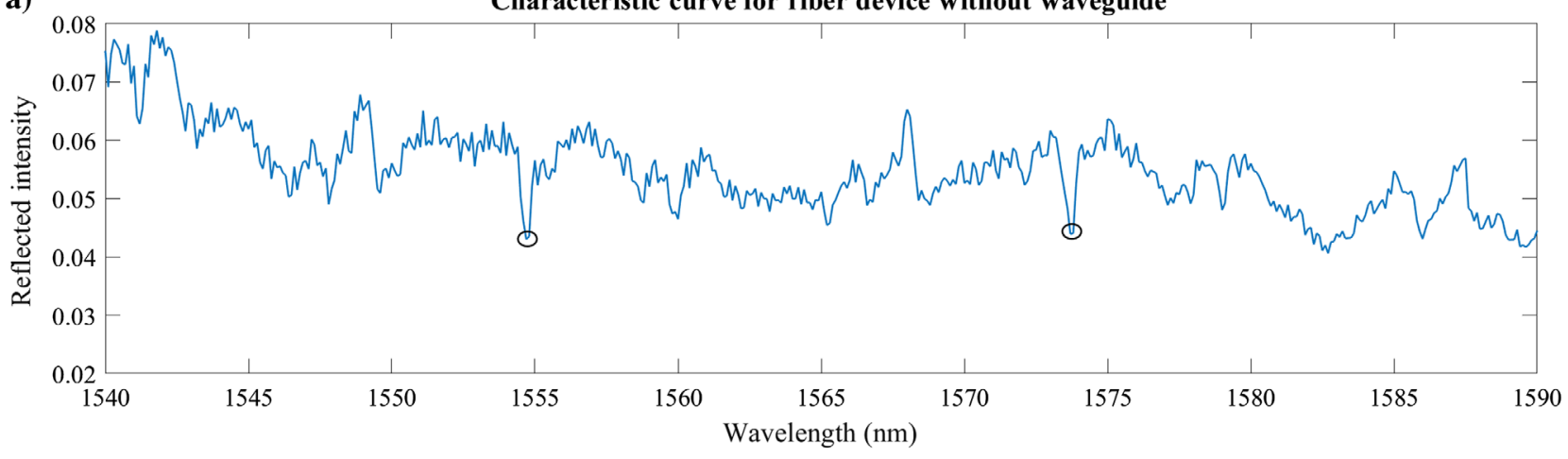

b)

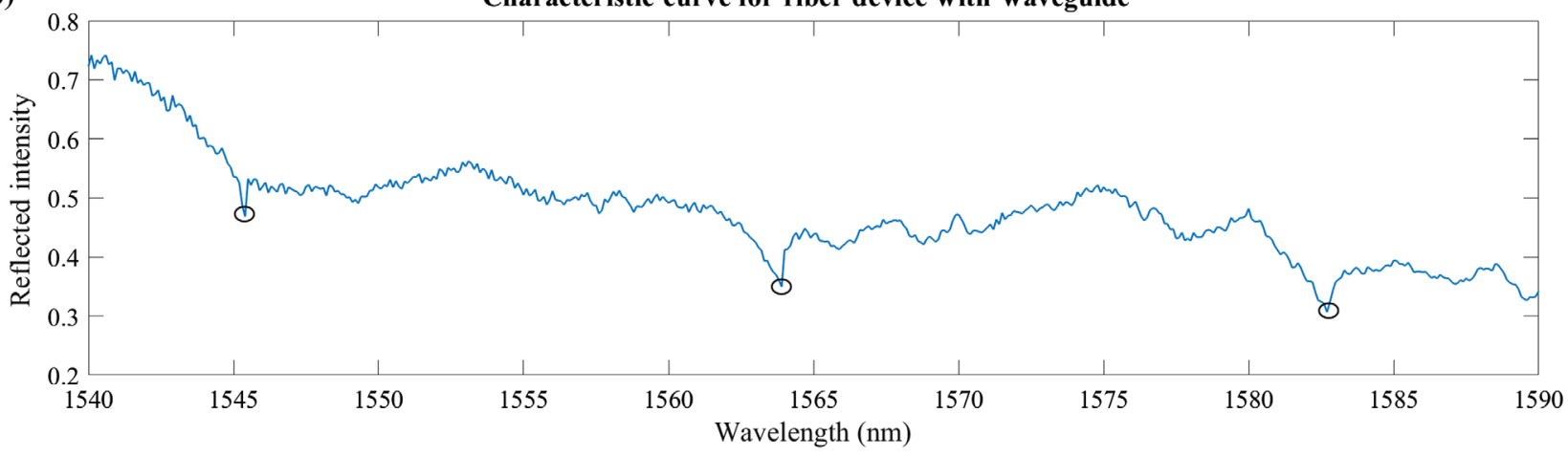

Fig. 11 Characteristic curves of the device (top) without waveguide and (bottom) with waveguide. Encircled points represent the resonant peaks.

this device were found to be 5139.80 and 62.79 , respectively. The bottom graph shows the improved sensitivity in a device fabricated including a waveguide. In this case, the $Q$-factor and finesse are calculated to be 7728.50 and 93.94 , respectively. Although these data do show a slight increase in the case of the device with waveguide, the sensitivity can be vastly improved if the nonideal shape of the pillar is addressed. Methods to do so are described in the following sections.

\subsection{Fiber-Ferrule Device Fabrication}

The $Q$-factor, and therefore sensitivity, of the interferometer increases with greater thicknesses as seen in Fig. 4. However, it is also desirable to have a thin device in order to improve the sensing bandwidth, leading to a trade-off between these parameters for an optimal design of the ultrasound detector. To fabricate resonator mirrors that are as flat and parallel to each other as possible, a method is proposed that employs a glass ferrule to hold the fiber. This increases the surface area available for dip coating of the cladding and thus offers control over the curvature of the cladding and the second mirror. This process was developed to obtain the dimensions for the model presented in Fig. 8, as well as to confirm the feasibility of depositing mirrors with gentler curvature than a hemispherical droplet.

Figure 12 shows the fabrication method proposed. First the $125-\mu \mathrm{m}$ bare fiber is inserted into a glass ferrule with inner diameter $125 \mu \mathrm{m}$ and outer diameter $1.8 \mathrm{~mm}$ and secured using UV-cured epoxy. The fiber surface is above the ferrule surface as shown in Fig. 12(a). Thereafter, the first mirror is deposited using e-beam evaporation. The gold-coated fiber tip is then dipped into the high-refractive index epoxy used to manufacture the waveguiding pillar resulting in a droplet as shown in Fig. 12(b). The dipped fiber is then exposed to 405-nm UV light through the back connector, and then washed in acetone to remove the uncured epoxy. This results in the waveguiding pillar as depicted in Fig. 12(c). To form the cladding, the combination is dipped into the cladding epoxy to the level of the ferrule, as shown in Fig. 12(d). This results in a droplet with a certain contact angle, depending on the epoxy. As an example, the ferrule shown in Fig. 13(b) resulted in a contact angle of $37 \mathrm{deg}$ when dipped in epoxy stereolithography (SLA) 3-D printer resin. Since this curve is spread over a relatively large area of diameter $1.8 \mathrm{~mm}$ as compared to the $125-\mu \mathrm{m}$ diameter of the fiber, the portion of the mirror above the fiber is almost parallel to the first mirror-meeting this important requirement for the waveguide device as described above.

As seen in Fig. 12(d), the distance between the tip of the pillar and the second mirror can be varied depending on how far beyond the surface of the ferrule the fiber is placed and secured. As shown in Fig. 8, it is observed that the distance between the tip of the fiber and the second mirror significantly affects the $Q$-factor.

Since the goal is to fabricate this device at the smallest possible size scale, it would be desirable to separate the fiber from the ferrule once the device has been completed. There are several ways this can be done:

1. The device can be made on a double clad fiber inserted into the ferrule. In this case, the cladding of the device can be selectively cured using the outer cladding of the fiber, allowing the use of the ferrule to achieve the desired contact angle but still separate the device after fabrication. 
(a)

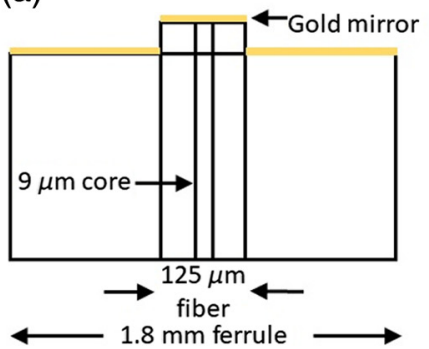

(b)

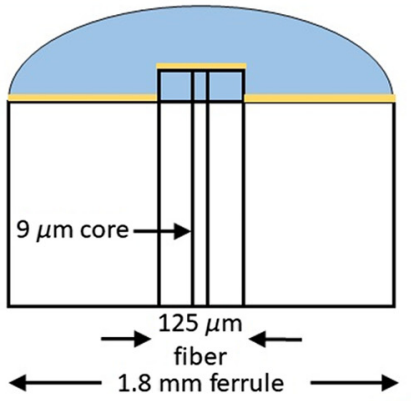

(c)

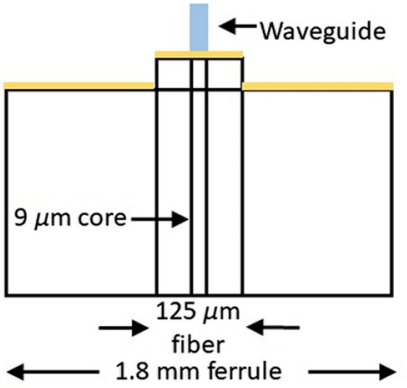

(d)

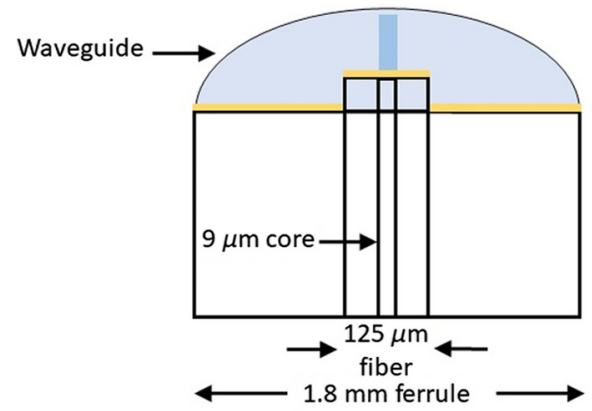

(e)

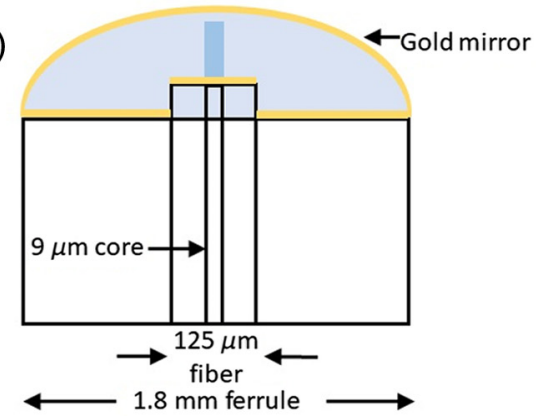

Fig. 12 Fabrication process proposed for fiber-ferrule devices: (a) first mirror deposited on fiber affixed to ferrule, (b) dip coating in waveguide epoxy, (c) pillar obtained by selective curing, (d) dip coating in cladding epoxy, and (e) deposition of second mirror.

(a)

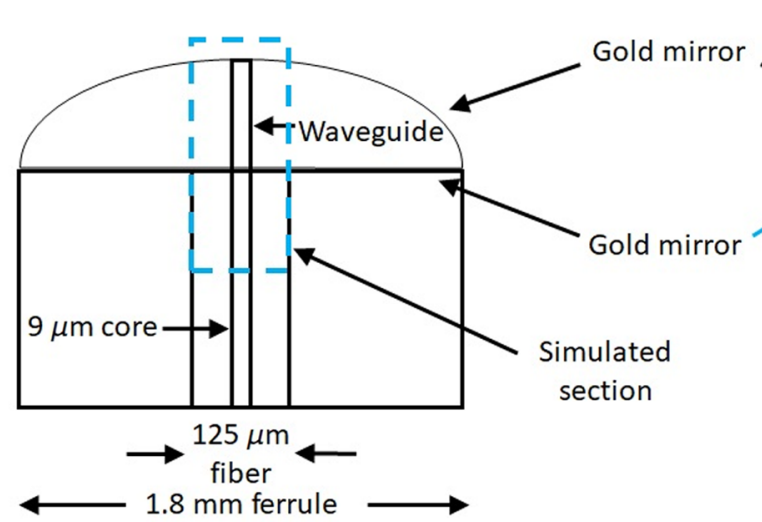

(b)

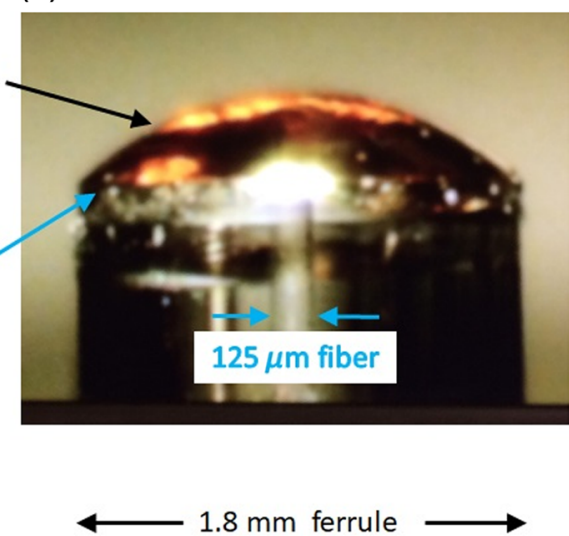

Fig. 13 Fiber-ferrule assembly highlighting (a) the simulated section and (b) a photograph of the same used for contact angle estimation.

2. The portion of the device directly above the fiber can be selectively cured from above using a mask/focused exposure.

\subsection{Nonideal Pillar Shape and Refractive Index Tuning}

The greatest challenge in our work so far has been to control the shape of the epoxy pillar produced, which serves as the waveguide. A perfectly flat waveguide is essential in order to preserve the flat wave front emerging from the fiber core. To understand the process of UV-curing within the polymer, two sets of experiments were conducted. First, the hemispherical drop of epoxy on the fiber tip resulting from dip coating was exposed to UV light through the fiber connector while varying the duration of exposure. The fibers were then washed in acetone, and the resulting pillars imaged under the microscope. As seen in Fig. 14, the pillars obtained from shorter duration exposures are seen to have a sharp, pointed tip, rather than the desirable cylindrical shape, and a larger mass appears to cure on top of this pointed pillar when exposure time is increased to $60 \mathrm{~s}$. To better understand the shapes of the pillars produced, the study was repeated while keeping the fiber tip immersed in a bulk volume of the epoxy during curing and washing in acetone thereafter. As expected, the shapes obtained reflected complex geometries.

A possible reason for this could be because the UV source used to cure the epoxy was broadband, containing wavelengths from 320 to $500 \mathrm{~nm}$, and the fiber is not a single-mode conductor for UV wavelengths. In addition, the curing of the epoxy is a dynamic process, in which portions of the epoxy that get cured first decide the path for UV light reaching the volume beyond. Therefore, it was desirable to use longer wavelengths so that 

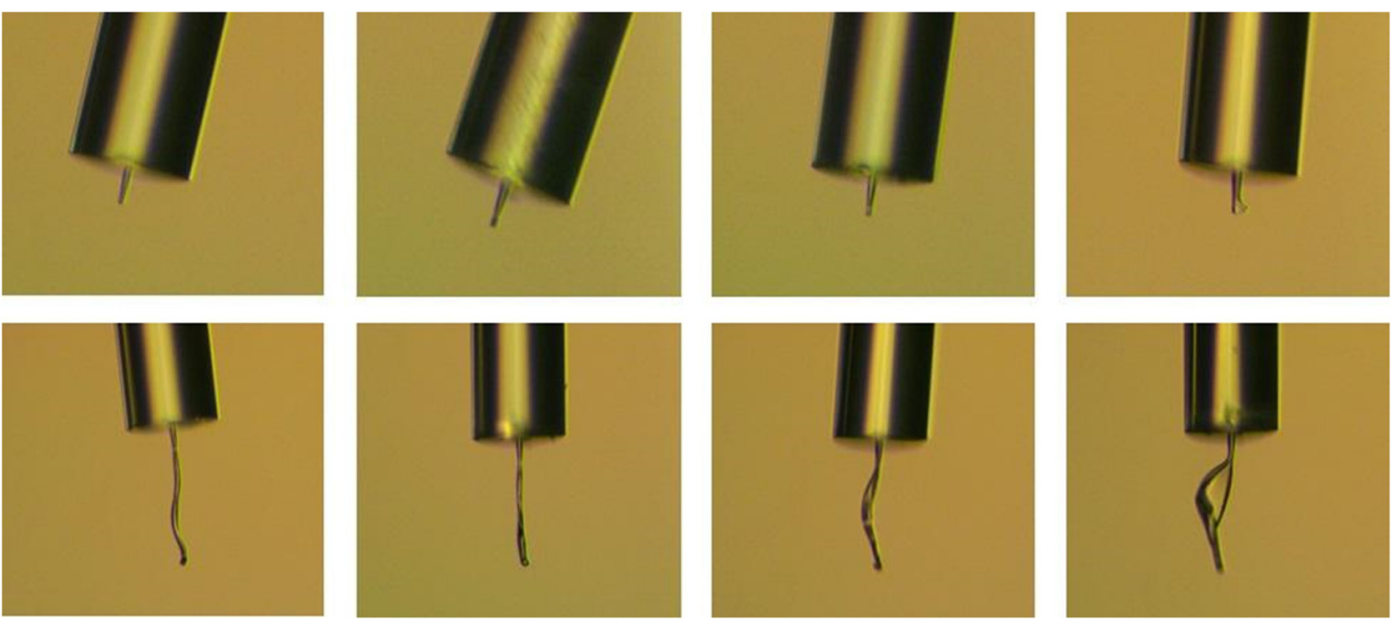

Fig. 14 Comparison of epoxy pillars resulting from (top row) droplet cure and (bottom row) bulk cure for cure times of $30 \mathrm{~s}, 60 \mathrm{~s}, 2 \mathrm{~min}$, and $5 \mathrm{~min}$.
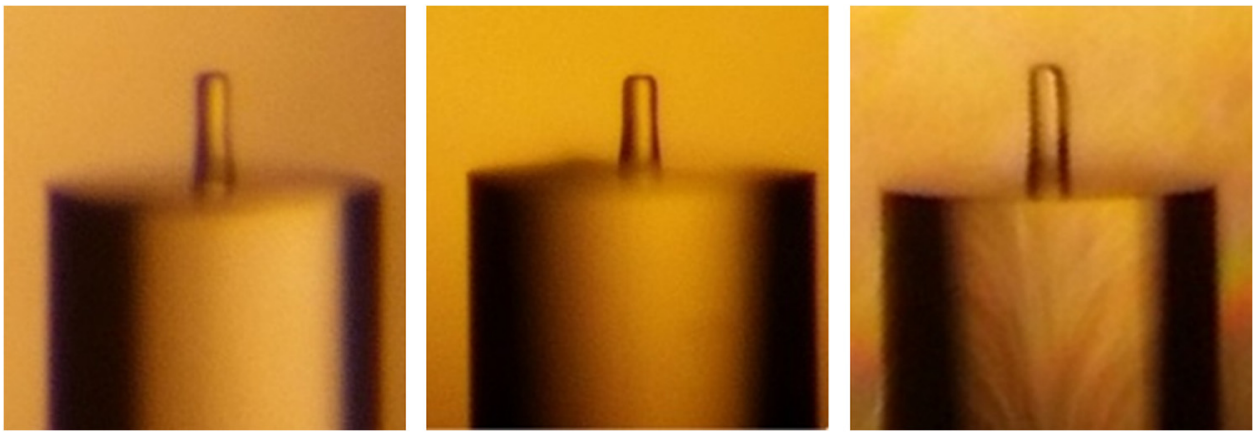

Fig. 15 Photographs of straight pillars obtained using 405-nm curing and multiple samples demonstrate repeatability of the process.

their penetration depth is greater than the device thickness, eliminating the complications resulting from progressive curing of epoxies at different depths. Another possibility was to change the epoxy being used since the photochemistry of the epoxy and the photoinitiator also affect the curing process. To test this, fibers were dip-coated in different epoxies and cured using a 405-nm laser through the back connector, and then dipped in acetone to wash away uncured epoxy. It was seen that epoxy SLA 3-D printer resin, designed to cure at $405 \mathrm{~nm}$ UV produced pillars that were consistently seen to be straight, perpendicular to the fiber surface, and with flat tops, satisfying our most important requirement. This is seen in Fig. 15.

This experiment confirmed that it was possible to fabricate epoxy waveguides with flat top surfaces by selective curing of UV sensitive epoxies, thus overcoming our biggest challenge. This waveguide structure can be incorporated into either bare fiber devices or fiber-ferrule assemblies as described above. Further, since the waveguide is self-aligned, this fabrication method can easily be extended to fiber bundles as well. Future work shall include complete characterization-optical and acoustic performance-of the devices fabricated with the waveguide and a cladding surrounding the waveguide with the requisite refractive index difference between the two materials for single-mode operation $(\sim 0.006$, calculated theoretically and confirmed via simulations), as well as dielectric mirrors for improved reflectivity.

\section{Discussion}

A method for fabricating all-optical US detectors is presented. This high finesse all-optical US transducer would allow forward-viewing at the size scale suitable for intravascular imaging. A method for creating waveguides to suppress diffraction losses is presented. By incorporating a waveguide into the structure of this device, the lateral power losses incurred can be minimized, improving the $Q$-factor, and therefore, the acoustic sensitivity of the device. Initial experimental results presented a challenge in obtaining straight polymer waveguides with flat top surfaces-essential to preserve the flat wave fronts that would emerge from them. Subsequently, through using longer wavelengths and experimenting with different photosensitive epoxies, experimental results are presented, which demonstrate the successful fabrication of straight, flat waveguides on the fiber substrate. With the help of simulations, a fabrication plan has been developed for completing the entire device. This fabrication method is suitable for use on fiber bundle devices for imaging and also allows for scaling in thickness to optimize detection bandwidth. Future work includes complete device fabrication and characterization to estimate the optical and acoustic sensitivity, NEP, and sensing bandwidth of the device. Such a device could be incorporated into either needle-based sensors to guide biopsies, or standard guidewires used in interventional cardiology for combined IVUS and photoacoustic sensing/imaging - a useful tool to address a range of clinical problems 
including interventions for CTO, which are an important and open clinical challenge.

\section{Disclosures}

The authors have no conflicts of interest to disclose.

\section{Acknowledgments}

The authors would like to acknowledge the funding received from the NSF and Institute for Engineering in Medicine (IEM) at the University of Minnesota. Portions of this work were conducted in the Minnesota Nano Center, which is supported by the National Science Foundation through the National Nano Coordinated Infrastructure Network (NNCI) under Award Number ECCS-1542202. We would like to thank Dr. Amin Tadayon for very useful technical discussions and Cameron Motameni for assistance in the lab.

\section{References}

1. J. Sapontis and J. Hill, "The role of adjunctive imaging in chronic total occlusions," Interv. Cardiol. 5(5), 577-589 (2013).

2. N. S. Rao and A. Chandra, "Needle guides enhance tissue adequacy and safety of ultrasound-guided renal biopsies," Kidney Res. Clin. Pract. 37(1), 41-48 (2018).

3. R. N. Uppot, M. G. Harisinghani, and D. A. Gervais, "Imaging-guided percutaneous renal biopsy: rationale and approach," Am. J. Roentgenol. 194(6), 1443-1449 (2010).

4. B. F. Waller, C. A. Pinkerton, and J. D. Slack, "Intravascular ultrasound: a histological study of vessels during life. The new 'gold standard' for vascular imaging," Circulation 85(6), 2305-2310 (1992).

5. M. H. Yamamoto et al., "Evaluation of chronic total occlusions by combined multi-detector computed tomography and intravascular ultrasound imaging," J. Am. Coll. Cardiol. 65(10), A1778 (2015).

6. F. Yamanaka et al., "A new imaging method for percutaneous coronary intervention of chronic total occlusions: real time cardiac computed tomography and coronary angiography image registration," J. Am. Coll. Cardiol. 65(10), A1887 (2015).

7. E. L. Wallace and K. M. Ziada, "Intravascular-ultrasound assisted localization and revascularization of an ostial chronic total occlusion: utility of near-field and far-field imaging," J. Invasive Cardiol. 27(3), E37-E39 (2015).

8. K. Dan et al., "Case report coronary artery hematoma in a case of chronic total occlusion treated with intravascular imaging and a novel scoring balloon catheter," Int. J. Clin. Exp. Med. 9(1), 408-411 (2016).

9. Eagle Eye Platinum, "Eagle Eye Platinum ${ }^{\circledR,}$, pp. 4-5.

10. B. K. Courtney et al., "Innovations in imaging for chronic total occlusions: a glimpse into the future of angiography's blind-spot," Eur. Heart J. 29(5), 583-593 (2008).

11. T. R. Shrout et al., "Piezoceramics for high-frequency (20 to $100 \mathrm{MHz}$ ) single-element imaging transducers," IEEE Trans. Ultrason. Ferroelectr. Freq. Control 44(5), 1038-1048 (1997).

12. C. Sheaff and S. Ashkenazi, "A fiber optic optoacoustic ultrasound sensor for photoacoustic endoscopy," in Proc. of IEEE Ultrasonics Symp., pp. 2135-2138 (2010).

13. P. C. Beard, A. M. Hurrell, and T. N. Mills, "Characterization of a polymer film optical fiber hydrophone for use in the range 1 to $20 \mathrm{MHz}$ a comparison with PVDF needle and membrane hydrophones," IEEE Trans. Ultrason. Ferroelectr. Freq. Control 47(1), 256-264 (2000).
14. P. Morris, A. Hurrell, and P. Beard, "Development of a 50-MHz FabryPérot type fibre-optic hydrophone for the characterisation of medical ultrasound," Proc. Inst. Acoust. 28, 717-725 (2006).

15. C. Sheaff and S. Ashkenazi, "A polyimide-etalon thin film structure for all-optical high-frequency ultrasound transduction," IEEE Trans. Ultrason. Ferroelectr. Freq. Control 59(10), 2254-2261 (2012).

16. J. D. Hamilton, "High frequency optoacoustic arrays using etalon detection," IEEE Trans. Ultrason. Ferroelectr. Freq. Control 47(1), 160-169 (2000).

17. S. Ashkenazi et al., "Optoacoustic imaging using thin polymer Etalon," Appl. Phys. Lett. 86(13), 134102 (2005).

18. C. Sheaff and S. Ashkenazi, "Characterization of an improved polyimide-etalon all-optical transducer for high-resolution ultrasound imaging," IEEE Trans. Ultrason. Ferroelectr. Freq. Control 61(7), 1223-1232 (2014).

19. J. Li et al., "Highly sensitive optical microresonator sensors for photoacoustic imaging," Proc. SPIE 8943, 89430C (2014).

20. J. A. Guggenheim et al., "Ultrasensitive plano-concave optical microresonators for ultrasound sensing," Nat. Photonics 11(11), 714-719 (2017).

21. M. A. Tadayon, M. E. Baylor, and S. Ashkenazi, "Polymer waveguide Fabry-Pérot resonator for high-frequency ultrasound detection," IEEE Trans. Ultrason. Ferroelectr. Freq. Control 61(12), 2132-2138 (2014).

22. B. Wang et al., "Intravascular photoacoustic imaging of lipid in atherosclerotic plaques in the presence of luminal blood," Opt. Lett. 37(7), 1244-1246 (2012).

23. S. Emelianov, "Intravascular ultrasound and photoacoustic imaging," in 30th Annual Int. Conf. of the IEEE, Engineering in Medicine and Biology Society (EMBS 2008), , IEEE (2008).

24. T. Allen et al., "Photoacoustic imaging of lipid rich plaques in human aorta," Proc. SPIE 7564, 75640C (2010).

25. S. Ashkenazi et al., "High frequency optoacoustic transducers for ultrasonic and photoacoustic imaging," in Photoacoustic Imaging and Spectroscopy, L. V. Wang, Ed., pp. 223-238, CRC Press, Boca Raton, Florida (2009).

26. H. Varu, "The optical modelling and design of Fabry-Pérot interferometer sensors for ultrasound detection," pp. 1-236, PhD thesis, University College London (2014).

27. E. Z. Zhang and P. C. Beard, "A miniature all-optical photoacoustic imaging probe," Proc. SPIE 7899, 78991F (2011).

28. S. V. Thathachary, C. Motameni, and S. Ashkenazi, "An all-optical fiber optic photoacoustic transducer," Proc. SPIE 10488, 104880M (2018).

Supriya V. Thathachary received her bachelor's degree in electrical and electronics engineering from Visvesvaraya Technological University, Belgaum, India, in 2012, and her MS degree in electrical engineering from the Indian Institute of Technology Madras, Chennai, India, in 2014. Since September 2014, she has been working toward her $\mathrm{PhD}$ in biomedical engineering at the University of MinnesotaTwin Cities, Minneapolis, Minnesota, USA. Her research interests include using micro/nanofabrication techniques, fiber-optic devices, and imaging and sensing to guide medical interventions.

Shai Ashkenazi received his BSc degree in physics from the Technion Israel Institute of Technology, Haifa, Israel, in 1988, and his $\mathrm{PhD}$ in physics from the Weizmann Institute of Science, Rehovot, Israel, in 1997. He then worked for six years in research and development companies in the fields of ultrasonic and optical devices for medical applications. In 2003, he moved to the University of Michigan, Ann Arbor, Michigan, USA, as a research scientist in the field of optoacoustic imaging. In 2008, he joined the Biomedical Engineering Department at the University of Minnesota, where he is currently an associate professor. His research interests include biomedical photonics, optoacoustics, and ultrasound imaging. 